\title{
Efectividad de un programa de estimulación en la memoria auditiva inmediata, la comprensión lectora y la resolución de problemas en niños de segundo grado de primaria
}

Effectiveness of a stimulation program in the immediate auditory memory, reading comprehension and problem solving in children of second grade

JUAN YARINGAÑo L

Universidad Marcelino Champagnat, Lima, Perú

(RECIBIDO 28/03/2014, ACEPTADO 26/05/2014)

\begin{abstract}
RESUMEN
La investigación estudia el efecto de un programa de estimulación en la memoria auditiva inmediata, la comprensión lectora y la resolución de problemas en un grupo de alumnos de segundo grado de educación primaria. En su realización se empleó el método experimental con un diseño cuasi experimental con grupo control. Se trabajó con 52 niños de ambos sexos, de segundo grado de primaria, quienes conformaron dos grupos, uno experimental y otro de control. Los sujetos fueron evaluados con el Test de Memoria Auditiva Inmediata (MAI), el Test de Complejidad Lingüística Progresiva (CLP) y la Prueba de Comportamiento Matemático, antes y después de la aplicación del programa de estimulación. El grupo experimental fue sometido a 16 sesiones de un programa de estimulación. Los resultados mostraron que el programa demostró ser efectivo en la memoria auditiva inmediata y en el componente de memoria lógica, ya que el puntaje del grupo experimental superó en el postest al grupo control. Del mismo modo, el grupo experimental obtuvo puntajes superiores al grupo control en la comprensión lectora. Finalmente, no se hallaron diferencias significativas entre el grupo experimental y el control en la resolución de problemas.
\end{abstract}

Palabras clave: memoria auditiva inmediata, comprensión lectora y resolución de problemas, educación primaria

1 Profesor de la Universidad Marcelino Champagnat. Email: jyaringano@umch.edu.pe. 
EFECTIVIDAD DE UN PROGRAMA DE ESTIMULACIÓN EN LA MEMORIA AUDITIVA INMEDIATA, LA COMPRENSIÓN LECTORA Y ...

\begin{abstract}
The research studies the effect of a stimulation program in the immediate auditory memory, reading comprehension and problem solving in a group of second graders primary education in their implementation the experimental method was employed with a quasi- experimental design with group control. We worked with 52 children of both sexes, second grade, who formed two groups one experimental and one control . Subjects were assessed with the Test of Auditory Immediate Memory (MAI), the Test of Linguistic complexity Progressive (CLP ) and the Test of Mathematical Behavior before and after application of the stimulation program. The experimental group underwent 16 sessions of a stimulation program. The results showed that the program was effective in the immediate auditory memory and logical memory component, as the score of the experimental group in the post test exceeded the control group. Similarly, the experimental group outperformed the control group in reading comprehension scores. Finally no significant differences between experimental and control groups were found in problem solving
\end{abstract}

Keywords: immediate auditory memory, reading comprehension and problem solving, elementary education.

\title{
INTRODUCCIÓN
}

Los resultados en educación en el Perú son un área que representa uno de los mayores retos por superar por el Estado y la sociedad en general. A nivel nacional, se han examinado las capacidades de los alumnos de educación básica relacionadas con la comprensión de textos y matemáticas. Los resultados obtenidos ponen en evidencia bajos desempeños, en matemáticas y comprensión lectora. Las conclusiones de la Evaluación Censal 2012 (Ministerio de Educación, 2013) en estudiantes de segundo grado de primaria establecen que en comprensión lectora el $30.9 \%$ de los alumnos se encuentran en el nivel 2 o satisfactorio, lo cual implica que, al finalizar el grado, lograron los aprendizajes esperados. El $49.3 \%$ de los alumnos se ubican el nivel 1 o en proceso, lo que indica que, al finalizar el grado, no lograron los aprendizajes esperados. Finalmente un $19.8 \%$ de los alumnos se encuentran debajo del nivel 1, lo cual señala que no lograron los aprendizajes esperados. Además, en el área de matemática el $12.8 \%$ de los alumnos presenta nivel 2, 38.2\% obtiene nivel 1 y el $49.0 \%$ se encuentra debajo del nivel 1. Estos resultados afectan a estudiantes varones y mujeres de instituciones educativas urbanas y rurales, estatales y no estatales. Por otro lado, en términos de equidad, los estudiantes provenientes de niveles socioeconómicos desfavorecidos también mostraron resultados bajos en los aprendizajes evaluados y los estudiantes de las instituciones educativas ubicadas en entornos rurales obtienen resultados más bajos con respecto de aquellos que estudian en instituciones ubicadas en zonas urbanas. En líneas generales, con estos resultados, se puede establecer que casi la mitad de los alumnos de segundo grado de educación primaria solo son capaces de comprender textos cortos de vocabulario sencillo; en el caso de matemática 
la mayoría (49.0\%) solo establece relaciones numéricas sencillas en situaciones desprovistas de contexto.

Estos resultados nos permiten describir el logro de los objetivos educativos a partir de la evaluación de ciertas habilidades o conocimientos que se espera logren los alumnos, los cuales están planteados por el currículo de estudios de Educación Básica Regular. Sin embargo, no nos permiten conocer cuáles son las características del funcionamiento a nivel cognitivo de los estudiantes, la influencia del entorno social, los estilos de enseñanza docente, los textos y métodos de enseñanza en comprensión lectora, las diferencias de acuerdo a diferentes variables, pues, si el objetivo primordial es construir programas más eficientes en la enseñanza, requerimos construir un modelo explicativo sobre la relación e influencia de las diferentes variables implicadas en la comprensión lectora y matemática. Pero uno de los factores cognitivos implicados en el proceso de comprensión lectora y la resolución de problemas es la memoria en funcionamiento, pues permite al lector ir integrando coherentemente la información semántica proveniente de las oraciones sucesivas y, en interacción con el conocimiento previo, ir agregando progresivamente la nueva información a una representación del texto que se va construyendo (García et al., 2006). Por lo tanto, un enfoque integral sobre la comprensión lectora y resolución de problemas deberá iniciarse con el conocimiento de los procesos cognitivos que la sustentan. Además, García (2002) señala que la mejora en las capacidades de memoria repercute en la comprensión de textos, dado que la lectura descansa en una capacidad de memoria operativa general, mas solo en un sistema basado específicamente en el lenguaje, lo cual determina una relación entre memoria operativa, comprensión y razonamiento. Asimismo Alsina (2007) concluye que los niños con menos disponibilidad de recursos de memoria tienen menor rendimiento en las tareas asociadas al cálculo.

\section{MÉTODO}

El diseño propuesto es el cuasi experimental con grupo control. Este diseño se emplea en situaciones en las cuales es difícil o casi imposible el control de variables extrañas que pueden afectar el trabajo investigado. El tipo de diseño específico: Diseño de dos grupos equivalentes. Este diseño consiste en que una vez que se dispone de los dos grupos, se debe evaluar a ambos en la variable dependiente, luego a uno de ellos se aplica el tratamiento experimental y el otro sigue con las tareas o actividades rutinarias. La muestra estuvo constituida por 52 alumnos, pertenecientes a dos aulas de segundo grado de educación primaria de una institución educativa estatal perteneciente a la UGEL 5, de San Juan de Lurigancho. Distribuidos de la siguiente manera: 26 en el grupo experimental y 26 en el grupo control, asignándose igual número de hombres y mujeres en cada grupo. Los instrumentos de recolección de datos en la etapa de pre y postest fueron: test de memoria auditiva inmediata (MAl), test de memoria auditiva inmediata (MAl) y la prueba de comportamiento matemático (PCM). 
EFECTIVIDAD DE UN PROGRAMA DE ESTIMULACIÓN EN LA MEMORIA AUDITIVA INMEDIATA, LA COMPRENSIÓN LECTORA Y ...

\section{RESULTADOS}

A continuación se presentan los resultados descriptivos antes y después de aplicar el programa experimental.

Figura 1. Variación de las medias en memoria auditiva inmediata del grupo experimental y control, antes y después de la aplicación del programa de estimulación

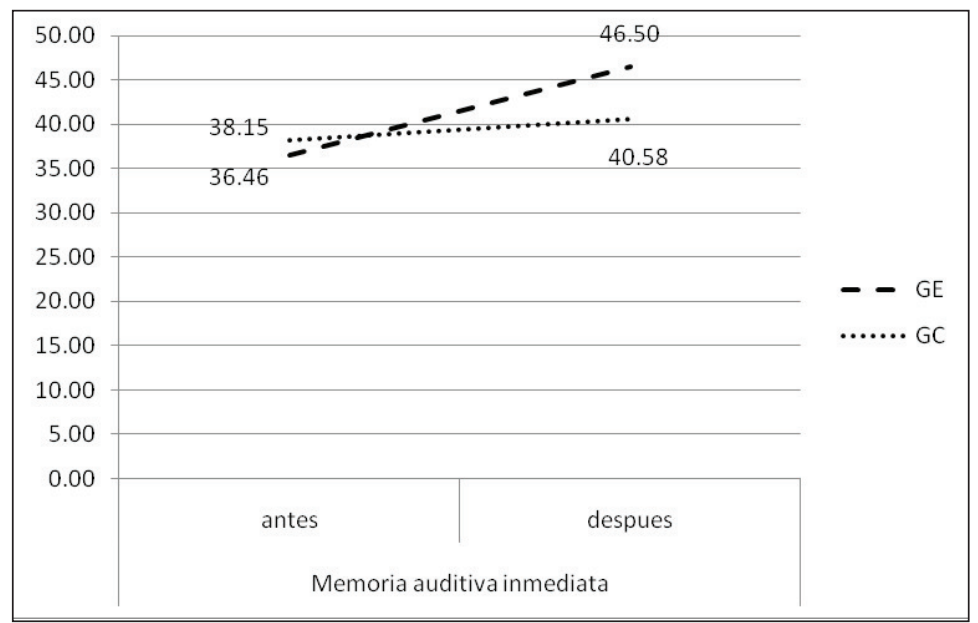

$\mathrm{Al}$ analizar la figura 1, las medias obtenidas en memoria auditiva inmediata por los grupos control y experimental. Se observó que en el postest el grupo experimental varía su puntaje promedio de 36.46 a 46.50 y en el grupo control de 38.15 a 40.58 .

Figura 2. Variación de las medias en los componentes memoria lógica, numérica y asociativa del grupo experimental y control, antes y después de la aplicación del programa de estimulación

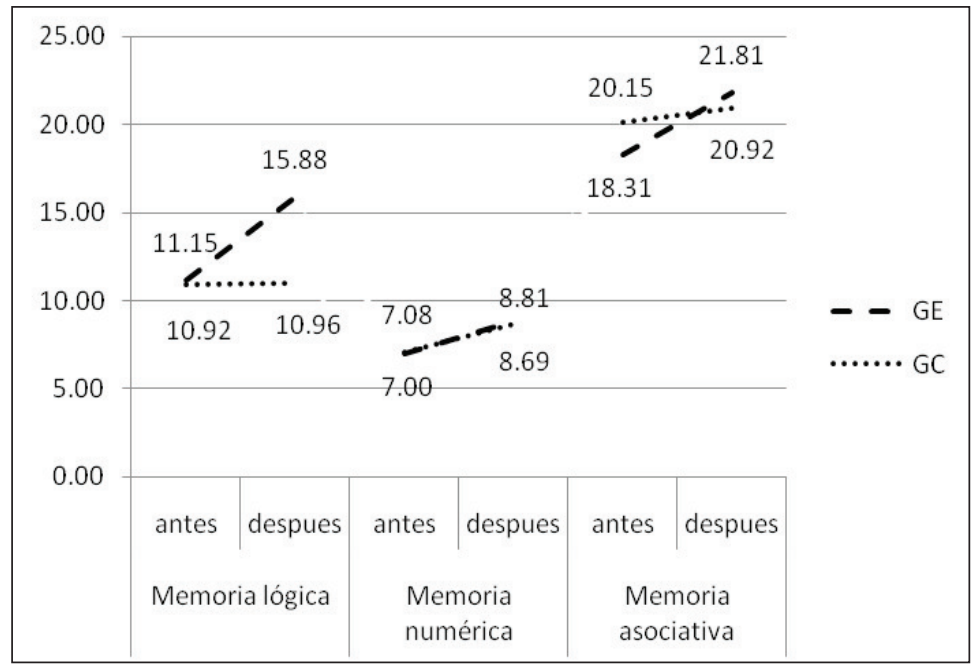


$\mathrm{Al}$ analizar la figura 2, las medias obtenidas en los grupos control y experimental se incrementan en el postest. En el caso de memoria lógica en el grupo experimental varía de 11.15 a 15.88 y en el grupo control de 10.92 a 10.96 ; en memoria numérica el grupo experimental varía su media de 7.00 a 8.81 y en el grupo control de 7.08 a 8.69; y en memoria asociativa el grupo experimental varía su media de 18.31 a 21.81 y en el grupo control de 20.15 a 20.92 .

Figura 3. Variación de las medias en comprensión lectora y resolución de problemas del grupo experimental y control, antes y después de la aplicación del programa de estimulación

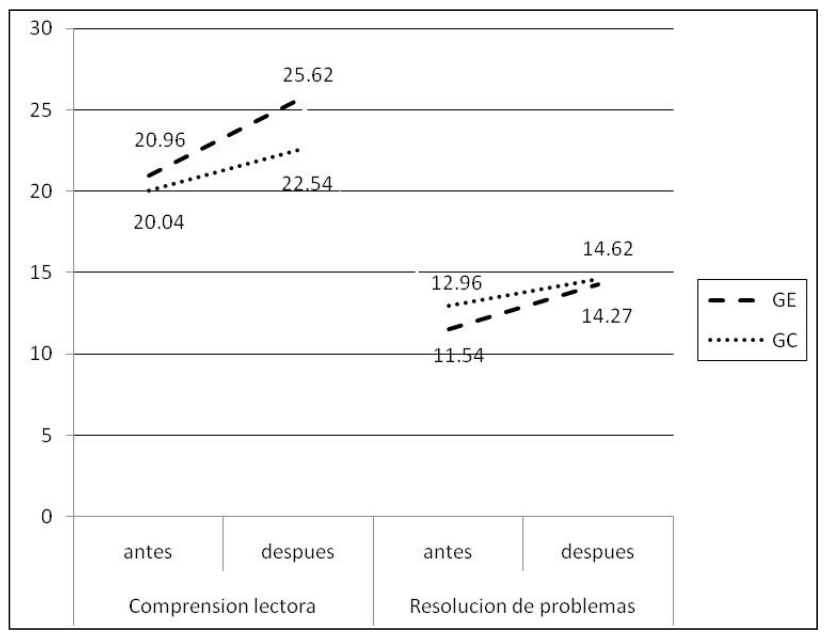

Según la figura 3, las medias obtenidas en los grupos control y experimental se incrementan en el postest. En el caso de la comprensión lectora en el grupo experimental varía de 20.96 a 25.62 y en el grupo control de 20.04 a 22.54. Por otro lado, en resolución de problemas el grupo experimental varía su media de 11.54 a 14.27 y en el grupo control de 12.96 a 14.62.

A continuación se presentan los resultados inferenciales antes y después de aplicar el programa experimental.

Tabla 1. Prueba de t para muestras independientes en grupo control y experimental, en memoria auditiva inmediata y sus componentes, comprensión lectora y resolución de problemas antes de la aplicación del programa de estimulación

\begin{tabular}{ccccc}
\hline & & $\mathrm{t}$ & $\mathrm{gl}$ & Sig. (bilateral) \\
\hline Memoria auditiva inmediata & -.521 & 50 & .605 \\
Componentes de & Memoria lógica & .279 & 50 & .782 \\
memoria auditiva inmediata & Memoria numérica & -.148 & 50 & .883 \\
& Memoria asociativa & -.779 & 50 & .440 \\
Comprensión lectora & & .844 & 50 & .403 \\
Resolución de problemas & & -1.797 & 50 & .078 \\
\hline
\end{tabular}

$\mathrm{N}=52$ 
En la tabla 1 se presentan los resultados de la prueba t para grupos independientes. Se observó que el estadístico $t$ en todos los casos tiene asociado niveles crítico bilaterales mayores a 0.05 , por lo tanto no podemos rechazar la hipótesis de igualdad de medias y concluir que las medias en comprensión lectora, resolución de problemas y memoria auditiva inmediata de niños del grupo control y experimental son similares. A raíz de estos resultados, podemos afirmar que ambos grupos pueden considerarse homogéneos.

Decisión: De acuerdo con los resultados de la tabla 5, se comprueba la hipótesis específica 1, y se concluye que no existen diferencias significativas en memoria auditiva inmediata y sus componentes, comprensión lectora y resolución de problemas entre el grupo control y experimental en niños de segundo grado de primaria, antes de la aplicación del programa de estimulación.

Tabla 2. Prueba de t para muestras independientes en grupo control y experimental, en memoria auditiva inmediata y sus componentes, después de la aplicación del programa de estimulación

\begin{tabular}{ccccc}
\hline & $\mathrm{t}$ & $\mathrm{gl}$ & \multicolumn{1}{c}{ Sig. (bilateral) } \\
\hline \multirow{2}{*}{ Memoria auditiva inmediata } & 2.030 & 50 & $.048^{*}$ \\
& Memoria lógica & 5.615 & 50 & $.000^{*}$ \\
Componentes de & Memoria numérica & .186 & 50 & .853 \\
memoria auditiva inmediata & Memoria asociativa & .463 & 50 & .645 \\
\hline
\end{tabular}

$\mathrm{N}=52$.

$* \mathrm{p}<0.05$

En la tabla 2 se presentan los resultados de la prueba t para grupos independientes. Se observó que el estadístico t en los casos de memoria auditiva inmediata y el componente memoria lógica tienen asociados niveles crítico bilaterales menores a 0.05 , por lo tanto podemos rechazar la hipótesis de igualdad de medias y concluir que en memoria auditiva inmediata y memoria lógica se presentan diferencias significativas entre el grupo experimental y el control.

Decisión: De acuerdo con los resultados de la tabla 6, se comprueba la hipótesis específica 2, y se concluye que existen diferencias significativas en memoria auditiva inmediata y el componente memoria lógica, entre el grupo control y el experimental en niños de segundo grado de primaria, después de la aplicación del programa de estimulación.

Tabla 3. Prueba de t para muestras independientes en grupo control y experimental, en comprensión lectora, después de la aplicación del programa de estimulación.

\begin{tabular}{lccc}
\hline & $\mathrm{t}$ & $\mathrm{gl}$ & Sig. (bilateral) \\
\hline \multicolumn{1}{c}{ Comprensión lectora } & 2.521 & 50 & .015 \\
\hline $\mathrm{N}=52$. & & & \\
$* \mathrm{p}<0,05$ & & &
\end{tabular}


En la tabla 3, se presentan los resultados de la prueba t para grupos independientes. Se observó que el estadístico t en el caso de comprensión lectora tiene asociado un nivel crítico bilateral menor a 0.05 , por lo tanto podemos rechazar la hipótesis de igualdad de medias y concluir que en comprensión lectora se presentan diferencias significativas entre grupo experimental y control.

Decisión: De acuerdo con los resultados de la tabla 7, se comprueba la hipótesis específica 3, y se concluye que existen diferencias significativas en comprensión lectora, entre el grupo control y el experimental en niños de segundo grado de primaria, después de la aplicación del programa de estimulación.

Tabla 8. Prueba de t para muestras independientes en grupo control y experimental, en resolución de problemas, después de la aplicación del programa de estimulación

\begin{tabular}{lccc}
\hline & $\mathrm{t}$ & $\mathrm{gl}$ & Sig. (bilateral) \\
\hline \multicolumn{1}{c}{ Resolución de problemas } & -.429 & 50 & .670 \\
\hline $\mathrm{N}=52$ & & & \\
${ }^{\mathrm{p}}<0.05$ & & &
\end{tabular}

En la tabla 8, se presentan los resultados de la prueba t para grupos independientes. Se observó que el estadístico t en el caso de comprensión lectora tiene asociado un nivel crítico bilateral mayor a 0.05 , por lo tanto no podemos rechazar la hipótesis de igualdad de medias y concluir que en resolución de problemas no se presentan diferencias significativas entre grupo experimental y control.

Decisión: De acuerdo con los resultados de la tabla 7, no se comprueba la hipótesis específica 4, y se concluye que no existen diferencias significativas en resolución de problemas, entre el grupo control y el experimental en niños de segundo grado de primaria, después de la aplicación del programa de estimulación.

\section{DISCUSIÓN}

Al analizar los resultados obtenidos, en el grupo experimental las medias obtenidas antes y después de la aplicación del programa experimental se observa un incremento en las puntuaciones en comprensión lectora y resolución de problemas, además la desviación estándar decrece. Esto indicaría que la estimulación de funciones cognitivas básicas, como la atención y la memoria, inciden en una mejora de las competencias escolares y a la homogeneización del grupo en sus resultados. Este incremento se explicaría en lo señalado por Aviles et al. (2006): "las alteraciones en las funciones cognitivas, como percepción, comprensión del lenguaje, memoria, abstracción y planificación, se reflejan en diversas dificultades de aprendizaje para la realización de tareas de lectoescritura y matemáticas".

Al comparar estos resultados con los del grupo control, también se observa un incremento en las medias obtenidas en comprensión lectora y resolución de problemas, pero no en la misma medida que el grupo experimental. Además los 
puntajes presentan una mayor dispersión. Estos datos se explican por el paso del tiempo y la madurez de los niños. Sobre esto, García et al. (1999) indican que, desde una perspectiva evolutiva, la limitación de las capacidades atencionales y de memoria es un factor mediador importante en el desarrollo de otras funciones cognitivas, esto debido a limitaciones de carácter estructural y que a medida que madura el cerebro de los niños les permite el afrontamiento de tareas cada vez más demandantes. Pero el hecho de que los puntajes en el grupo experimental estén más agrupados en torno a la media de los del grupo control podría indicar que el programa de estimulación permite un desarrollo y madurez más homogéneo en las capacidades cognitivas básicas.

Las hipótesis planteadas en el presente estudio tratan de conocer la efectividad del programa de estimulación en la memoria auditiva inmediata, la comprensión lectora y la resolución de problemas. De este modo, la primera hipótesis buscó conocer los niveles en estas variables al inicio del programa, con lo cual se comprueba que, antes de aplicar el programa de estimulación, los sujetos del grupo control y experimental no presentan diferencias significativas en sus puntajes medios, al observarse detenidamente las dos primeras variables. La estrategia utilizada por los niños para almacenar y recuperar información es repetir sistemáticamente la información recibida. Sobre esto, Kail (1990) indica que los niños de 7 años tienden a utilizarla en las distintas actividades que demandan un esfuerzo cognitivo, y dado que es la primera estrategia que utilizan, tiende a generalizarse, siendo rígida y poco flexible.

Pero al finalizar el programa de estimulación, se observa que el grupo experimental y el grupo control presentan diferencias significativas en las puntuaciones en memoria lógica y comprensión lectora. Por el contrario, no se presentan diferencias significativas en los puntajes de resolución de problemas después de la aplicación del programa de estimulación. Al tratar de conocer la efectividad del programa, se planteó como hipótesis conocer si existen diferencias significativas en los incrementos entre el grupo experimental y el grupo control. Los resultados señalan que los incrementos son significativos a favor del grupo experimental en los puntajes de comprensión lectora, resolución de problemas, memoria lógica y memoria auditiva inmediata, después de la aplicación del programa de estimulación. Por lo tanto, podemos afirmar que el programa de estimulación es efectivo en estas áreas, pero no lo es en el caso de memoria numérica y asociativa. Estos resultados son similares a los obtenidos por Álsina y Saez (2004), quienes aplicaron un programa de estimulación de la memoria para niños de 7 y 8 años. En la evaluación postest sus resultados indican que, aunque todos mejoran su memoria de trabajo, los niños del grupo experimental presentan incrementos estadísticamente significativos, siendo la repercusión más significativa en las tareas de administración de los recursos de memoria asociados al componente ejecutivo central. 


\section{CONCLUSIONES}

Los resultados obtenidos en el presente estudio indican que es posible entrenar las capacidades asociadas a la memoria en niños. De modo más específico, los resultados indicaron que el programa administrado tiene repercusiones en las funciones asociadas a los procesos atencionales de selección, distribución y mantenimiento de la actividad psicológica; esto permitiría un mejor uso de los recursos cognitivos asociados a la memoria auditiva inmediata, lo cual determinaría incrementos significativos en las capacidades de memoria. El presente estudio también permite concluir que la estimulación de procesos cognitivos básicos incide en los niveles de comprensión lectora y resolución de problemas matemáticos en estudiantes de educación primaria.

\section{REFERENCIAS BIBLIOGRÁFICAS}

Alsina, A. (2003) ¿Es posible entrenar la memoria de trabajo?: un programa para niños de 7-8 años. Infancia y Aprendizaje, 27 (3), 275-287.

Alsina, A. (2007) ¿Por qué algunos niños tienen dificultades para calcular? una aproximación desde el estudio de la memoria humana Revista Latinoamericana de Investigación en Matemática Educativa, noviembre, año/ vol. 10, número 003, pp. 315-333.

Ardila, A. y Russelli, M. (2000). Neuropsicología clínica. Prensa creativa, p. 34.

Avilés, M.; Flórez, L. y Patiño, M. (2006) Caracterización cognitiva de los estudiantes con dificultades de aprendizaje de la Institución Educativa 26 de Marzo, de Barrancabermeja. Pensando Psicología, Revista de la Facultad de Psicología Universidad Cooperativa de Colombia, Vol. 3, No 3 .

Baddeley, A. D. (1998) Memoria Humana. Teoría y Práctica. Madrid: McGraw-Hill.

Baddeley, A.D., \& Hitch, G. (1974). Working memory. In G.H. Bower (Ed.), The psychology of learning and motivation: Advances in research and theory (Vol. 8, pp. 47--89). New York: Academic Press.

Cuetos, F. (1990). Psicología de la escritura. Madrid: Escuela Española.

Cuetos, F. (1991). Psicología de la lectura. Madrid: Escuela Española.

Gutiérrez, F. García J.; Elosúa R.; Luque J. y Garate, M (2002) Memoria operativa y comprensión lectora: algunas cuestiones básicas. Acción Psicológica 1, 45-68.

García, J. (2006) Lectura y conocimiento. Barcelona: Paidós.

García, J.; Elosúa, M.; Gutiérrez, F.; Luque J. y Garate, M (1999) Comprensión lectora y memoria operativa. Barcelona: Paidós.

García, J.; Martín, J.; Luque, J. y Santamaría, C. (1995) Comprensión y adquisición de conocimientos a partir de textos. Madrid: Siglo XXI. 
EFECTIVIDAD DE UN PROGRAMA DE ESTIMULACIÓN EN LA MEMORIA AUDITIVA INMEDIATA, LA COMPRENSIÓN LECTORA Y ...

García, E. M. (1998). ENFÓCATE. Programa de entrenamiento en focalización de la atención. Bilbao:Grupo Albor-Cohs.

García, J. y Gonzáles, D. (2003). Cuaderno para mejorar memoria y atención I: nivel óptimo, $1^{\mathrm{o}}$ y $2^{\circ}$ ciclo de primaria. Madrid: EOS.

García-Sevilla, J. (2006). Mejorar la atención en el niño. Madrid: Pirámide.

Kail, R. (1990) El desarrollo de la memoria en los niños. Madrid: Siglo XXI de España Editores, $2^{\mathrm{a}}$ ed.

Klingberg, T., Forssberg, H. \& Westerberg, H. (2002). Training of working memory in children with adhd. Journal of clinical and experimental neuropsychology, 24 (6), 781-791.

Landa, L. (2004) El déficit mnésico en niños con problemas de aprendizaje. Un estudio en población infantil peruana. En Cuadros, E.; da Silva, A.; Paz, C. y Mena, J. (Eds.) II Congreso internacional de científicos peruanos (p. 178). Lima: Red mundial de científicos peruanos.

Unidad de Medición de la Calidad Educativa (2013) Programa para la Evaluación Internacional de Estudiantes (PISA). Primeros resultados PISA 2012. Lima MINISTERIO DE EDUCACIÓN.

Unidad de Medición de la Calidad Educativa (2013) Resultados de la Evaluación Censal de Estudiantes 2012 (ECE 2012). Lima MINISTERIO DE EDUCACIÓN.

Miranda-Casas, A.; Presentación-Herrero, M. y Gargallo-López B. (2000) Intervención psicoeducativa en estudiantes con trastorno por déficit de atención con hiperactividad. Revista de Neurología Clínica; 1: 203-216.

Montealegre, R. y Forero, L. (2006) Desarrollo de la lectoescritura: adquisición y dominio. En: Acta colombiana de psicología, 9(1), 25-40.

Nairne, J.S. (1988). A framework for interpreting recency effects in immediate serial recall. Memory \& Cognition, 16, 343-352.

Nairne, J.S. (1990) A feature model of inmediate memory. Memory and cognition, 18 (3), 251-269.

Nairne, J.S., \& Mcnabb, W. K. (1985). More modality effects in the absence of sound. Journal of Experimental Psychology: Learning,Memory, \& Cognition, 11, 596-604.

Nairne, J. S., \& Walters, V. L. (1983). Silent mouthing produces modality- and suffix-like effects. Journal of Verbal Learning \& Verbal Behavior, 22, 475-483.

Pólya, G. (1990). Cómo plantear y resolver problemas. México: Trillas

Smith, E. \& Jonides, J. (1998) Neuroimaging analyses of human working memory. En: Proc. Natl. Acad. Sci. USA. Vol. 95, pp. 12061-12068, September. 


\section{ANEXO}

\section{PROGRAMA DE ESTIMULACIÓN APRENDER SIN PROBLEMAS}

\section{NIVEL 1: Atención y Memoria}

\section{DESCRIPCIÓN GENERAL}

$\begin{array}{ll}\text { Autor } & \text { : Juan Yaringaño Limache } \\ \text { Lugar } & \text { : Lima } \\ \text { Dirigido a } & : \text { Niños de educación primaria de EBR } \\ \text { Duración } & : \text { 16 sesiones, de } 45 \text { minutos cada una } \\ \text { Objetivo } & : \text { Estimular las funciones cognitivas de atención y memoria } \\ \text { Estrategias } & \text { : Entrenamiento cognitivo y técnicas cognitivo-conductuales }\end{array}$

\section{OBJETIVOS GENERALES}

Mejorar los niveles de atención y memoria auditiva en ambientes escolares y familiares.

Disminuir los niveles de actividad excesiva y déficit de atención.

\section{OBJETIVOS ESPECÍFICOS}

Incrementar las habilidades para dar respuesta a un solo estímulo o tarea en presencia de otros estímulos o tareas.

Distribuir las conductas de atención utilizadas cuando el ambiente nos exige atender varias cosas a la vez.

Mantener y sostener la atención por períodos de tiempos mayores cuando se realiza una sola tarea.

Disminuir los niveles de actividad mediante el aprendizaje de técnicas de relajación.

Evocación de estímulos en material icónico y verbal.

Evocación de sonidos naturales o ambientales.

Evocación de palabras, frases o textos

\section{ESTRATEGIAS}

Considerar a la conducta de atención como una habilidad capaz de ser desarrollada mediante técnicas de reforzamiento positivo abierto y encubierto, modificaciones en el ambiente, el control de los eventos y de autoinstrucciones verbales. 
EFECTIVIDAD DE Un PROGRAMA DE ESTIMULACIÓN EN LA MEMORIA AUDITIVA INMEDIATA, LA COMPRENSIÓN LECTORA Y ...

\section{Destinadas a iniciar la atención}

Analizar los intereses básicos de los niños.

Desarrollar las conductas de orientación y reacción ante la presencia de un estímulo.

Detectar estímulos específicos, ya sea por su presencia o ausencia.

\section{Destinadas a mantener la atención}

Mantener la atención hacia un mensaje relevante, dejando de lado otros.

Evitar la interferencia de otros estímulos no importantes.

Desechar expectativas e ideas que interrumpan las tareas en curso.

\section{Destinadas a distribuir la atención}

Identificar las diferencias entre dos o más estímulos.

Identificar similitudes en función de un criterio previo.

Almacenar información presentada en diversas formas.

Reconocer material gráfico escrito previamente presentado.

Repetir información aprendida previamente en dos canales sensoriales distintos.

\section{Evocación de estímulos en material icónico y verbal}

Reproducir o dibujar estímulos previamente presentados

Describir verbalmente el material que se ha presentado.

Recuerdo del contenido de escenas complejas.

Reconocimiento de escenas faltantes o cambiantes en un relato, lámina o secuencia.

\section{Evocación de sonidos naturales o ambientales}

Reconocer uno o varios sonidos naturales entre otros que han sido presentados previamente al sujeto.

Repetir uno o varios sonidos naturales entre otros que han sido presentados previamente al sujeto, en el mismo orden o en forma aleatoria.

\section{Evocación de palabras, frases o textos}

Reconocer una o varias palabras, frases o textos entre otros que han sido presentados previamente al sujeto. 
Repetir una o varias palabras, frases o textos entre otros que han sido presentados previamente al sujeto, en el mismo orden o en forma aleatoria.

\section{SESIONES}

Sesión 1 : Relajación y seguimiento de instrucciones

Sesión 2 : Atención selectiva - Autoinstrucciones

Sesión 3 : Memoria auditiva y visual

Sesión 4 : Atención sostenida- Identificar y mantener

Sesión 5 : Memoria auditiva y visual

Sesión 6 : Atención dividida - Diferenciar estímulos

Sesión $7 \quad$ : Memoria auditiva y visual

Sesión 8 : Atención selectiva - Detección de señales

Sesión 9 : Atención sostenida - Seleccionar y aislar

Sesión 10 : Atención dividida - Ejecución de dos tareas

Sesión 11 : Atención total o integral

Sesión 12 : Memoria auditiva y visual

Sesión 13 : Atención total o integral

Sesión 14 : Memoria auditiva y visual

Sesión 15 : Atención total o integral

Sesión 16 : Memoria auditiva y visual 